\title{
Age estimation in the living: Transition analysis on developing third molars
}

\author{
Sara Tangmose ${ }^{\mathrm{a}, *}$, Patrick Thevissen ${ }^{\mathrm{b}}$, Niels Lynnerup ${ }^{\mathrm{a}}$, Guy Willems ${ }^{\mathrm{b}}$, Jesper Boldsen ${ }^{\mathrm{c}}$ \\ a Section of Forensic Pathology, Institute of Forensic Medicine, University of Copenhagen, Copenhagen, Denmark \\ ${ }^{\mathrm{b}}$ Forensic Dentistry, Department of Oral Health Sciences, KU Leuven E Dentistry, University Hospitals Leuven, Belgium \\ ${ }^{\mathrm{c}}$ Department of Anthropology (ADBOU), Institute of Forensic Medicine, University of Southern Denmark, Denmark
}

\section{A R T I C L E I N F O}

\section{Article history:}

Received 8 January 2015

Received in revised form 18 May 2015

Accepted 27 July 2015

Available online 5 August 2015

\section{Keywords:}

Third molars

Transition analysis

Age estimation in the living

Forensic Anthropology Population Data

\begin{abstract}
A B S T R A C T
A radiographic assessment of third molar development is essential for differentiating between juveniles and adolescents in forensic age estimations. As the developmental stages of third molars are highly correlated, age estimates based on a combination of a full set of third molar scores are statistically complicated. Transition analysis (TA) is a statistical method developed for estimating age at death in skeletons, which combines several correlated developmental traits into one age estimate including a 95\% prediction interval. The aim of this study was to evaluate the performance of TA in the living on a full set of third molar scores. A cross sectional sample of 854 panoramic radiographs, homogenously distributed by sex and age (15.0-24.0 years), were randomly split in two; a reference sample for obtaining age estimates including a 95\% prediction interval according to TA; and a validation sample to test the age estimates against actual age. The mean inaccuracy of the age estimates was 1.82 years $( \pm 1.35)$ in males and 1.81 years $( \pm 1.44)$ in females. The mean bias was 0.55 years $( \pm 2.20)$ in males and 0.31 years $( \pm 2.30)$ in females. Of the actual ages, $93.7 \%$ of the males and $95.9 \%$ of the females (validation sample) fell within the $95 \%$ prediction interval. Moreover, at a sensitivity and specificity of 0.824 and 0.937 in males and 0.814 and 0.827 in females, TA performs well in differentiating between being a minor as opposed to an adult. Although accuracy does not outperform other methods, TA provides unbiased age estimates which minimize the risk of wrongly estimating minors as adults. Furthermore, when corrected ad hoc, TA produces appropriate prediction intervals. As TA allows expansion with additional traits, i.e. stages of development of the left handwrist and the clavicle, it has a great potential for future more accurate and reproducible age estimates, including an estimated probability of having attained the legal age limit of 18 years.
\end{abstract}

(c) 2015 Elsevier Ireland Ltd. All rights reserved.

\section{Introduction}

The correlation between the degree of tooth mineralization and chronological age can be used for estimating chronological age in individuals where the age is unknown $[1,2]$. In most countries, the chronological age of 18 years mark the difference between being a minor and child as opposed to an adult [3]. Above the chronological age of approximately $14.0-16.5$ years, third molars are the only teeth left to mineralize [4,5]. Although highly variable, a radiographic assessment of third molar development is essential for differentiating between minors and adults in young asylum seekers without documents to prove their age $[2,3]$.

\footnotetext{
* Corresponding author at: Section of Forensic Pathology, Institute of Forensic Medicine, Frederik V's vej 11, 1st floor, 2100 Copenhagen Ø, Denmark. Tel.: +45 61333372 .

E-mail address: saral@sund.ku.dk (S. Tangmose).
}

The majority of reference studies investigating dental age report the correlation between chronological age and the stages of third molar development as means and standard deviations for the third molars individually: upper right (UR), upper left (UL), lower left (LL), and lower right (LR) [6-8]. Unfortunately, mean ages are affected by the age composition of the reference sample. This bias is known as age-mimicry [9]. Although small, when used for age estimation purposes this bias may affect whether the examinee is assessed as a child or an adult. For example, the mean age at stage $R_{3 / 4}$ in UR has been reported as both 17.0 years and 18.3 years $[8,10]$. Thus, simply taking the mean of means or using discrete age intervals may become attractive, although actually methodologically wrong.

A few published studies provide age estimates from combinations of a full set of third molar scores [1,11,12]. Unfortunately, such statistical models are complicated due to the correlation between the developmental stages of third molars, i.e. the developmental stage of one third molar carries information about 
the developmental stage of the other third molars. Consequently, correlation between biological traits limits the number which can be integrated in a classical regression model. Such examples can be found in Bassed et al. [13] and in Gunst et al. [1]. Moreover, due to a systematic bias (attraction of the middle), traditional regression models systematically overestimate the age of younger individuals and underestimate the age of older individuals [11]. The direction of this bias risks that minors are assessed as adults, which should be avoided [11].

Regressing biological traits on age, i.e. treating age as the explanatory variable, removes the bias of age-mimicry and the tendency to overestimate the age of young [14]. However, these estimates have to be manipulated to produce estimates of age [9]. For this purpose, the use of Bayes theorem has been proposed $[9,11,14]$. Bayes theorem states that the prior probability of a phenomenon should play a role in evaluating its posterior probability. Methods using Bayesian statistics for estimating age in the living have been published for third molars [11] and clavicles [15].

In lack of perfect correlation between a biological trait and age the Study Group on Forensic Age Diagnostics (AGFAD) and Forensic Anthropology Society of Europe (FASE) recommend to use as much information as possible, i.e. to combine several traits in order to achieve more accurate age estimates with appropriate levels of uncertainty [2,3]. Although this multi-factorial approach is recommended, no current method combine the traits used most in forensic age estimation cases (hand-wrist, teeth and clavicles) to obtain an estimate of age including a 95\% prediction interval [16]. While the Bayesian method proposed by Thevissen et al. showed promising results, it is computationally too complicated to allow for further traits to be added [11]. Transition analysis (TA) may be an important step towards a solution to this problem. TA is a Bayesian approach developed for assessing age-at-death in skeletons with the aim of avoiding age-mimicry [9]. It combines several developing age-related traits into one point age estimate including a $95 \%$ prediction interval. TA assumes conditional independence, i.e. that any correlation between traits is purely attributable to age. This assumption simplifies computations, allowing probabilities to be multiplied. Even when this assumption does not hold, TA still provides age estimates which are unbiased. However, confidence intervals appear narrower than they really are. This bias is corrected ad hoc. According to the originators, TA is applicable in any trait that changes unidirectional with age in nonoverlapping stages and always from stage $i$ to stage $i+1$. Restricting this approach to forensic odontology, this paper presents the first attempt to apply TA on radiological stage assessments of third molar development with the aim to provide age estimates including a $95 \%$ prediction interval from a full set of third molar scores.

\section{Materials and methods}

Statistical analyses were performed using data from a crosssectional sample comprising 854 panoramic radiographs of subjects in the chronological age range 15.0-24.0 years. All subjects comprised a full set of third molar scores. The sample was archived panoramic radiographs used for diagnosis and treatment planning collected at the Department of Oral Medicine and Oral Diagnosis, School of Dentistry \& Dental Research Institute, Seoul National University, Republic of Korea. For each radiograph nationality, birth date and gender was verified by use of the official birth certificate and (or) the identity card. The date of radiographic exposure was registered. The subjects had no history of medical diseases or interventions affecting the presence and/or development of teeth. Radiographs were scored in blind trials at random order by a forensic odontologist, using the ten-point

\section{Table 1}

Distribution of males and females in the two samples by age group. Age group defines the period between the date of the birthday until the day before the next

\begin{tabular}{llllll}
\hline \multirow{2}{*}{ Age group (years) } & \multicolumn{2}{l}{ Reference sample } & & \multicolumn{2}{l}{ Validation sample } \\
\cline { 2 - 3 } \cline { 5 - 6 } & Male $n$ & Female $n$ & & Male $n$ & Female $n$ \\
\hline 15 & 32 & 20 & & 19 & 26 \\
16 & 25 & 24 & & 29 & 29 \\
17 & 25 & 16 & & 15 & 26 \\
18 & 27 & 26 & & 32 & 28 \\
19 & 32 & 27 & & 24 & 25 \\
20 & 19 & 23 & & 22 & 25 \\
21 & 24 & 28 & & 15 & 29 \\
22 & 17 & 25 & & 32 & 12 \\
23 & 19 & 19 & & 17 & 21 \\
\hline
\end{tabular}

scoring system according to the method of Gleiser and Hunt, modified by Köhler [8]. Additionally, $10 \%$ of the radiographs were randomly selected and scored by a second investigator. The scorings were repeated after 1 month. Kappa statistics revealed no significant intra- or inter-observer effects. The Köhler technique was chosen as it provides an additional stage (open apex) for sub adult age estimation as compared to the Demirjian technique [17]. Although small in magnitude, the amount of stages have been shown to slightly influence the age predictions [18]. Stages $\leq 5$ were considered as one category to increase regression robustness.

A Wilcoxon Signed Rank test was used to test bilateral stage differences. Correlation between the four third molars was investigated by partial correlation, controlling for age.

The sample was randomly split in two, stratified by age and sex (Table 1 ). The reference sample, $n=428$ cases (220M, 208F) was used to develop the age estimates including the 95\% prediction intervals. The validation sample, $n=426(205 \mathrm{M}, 221 \mathrm{~F})$ was used to test the precision of the age estimates and the $95 \%$ prediction intervals.

Age estimates of third molar development were obtained according to the statistical approach of TA, described by fitted binary regressions known as the "continuation ratio model" [9]. In the age range 15.0-24.0 years, probability functions (3288 data points) for males and females were computed at each stage transition for the four third molars individually as described by Eqs. (5.5)-(5.7) in Boldsen et al. [9]. Maximum likelihood estimates of $\alpha$ and $\beta$ were obtained using a general linear model (glm) in the freely available statistical package "R" [19]. We assumed a uniform prior distribution to obtain estimates of age. Thus, the value of age which fulfills the maximum likelihood was considered the age estimate. The $95 \%$ prediction intervals for the age distribution were all ages with likelihoods greater than 0.1465 [20]. In order to avoid too optimistic predictions intervals, they were corrected ad hoc by replacing $\left(L\left(a \mid y_{j}\right)=i\right) /\left(L\left(\hat{a} \mid y_{j}\right)=i\right)$ in equation 5.14 [9] with $\left[\left(L\left(a \mid y_{j}\right)=i\right) /\left(L\left(\hat{a} \mid y_{j}\right)=i\right)\right]^{1 / 2}$, where $r=\operatorname{var}($ obs $) / \operatorname{var}($ est $)$, and $\operatorname{var}(-$ obs) is the observed variance of age (validation sample) and $\operatorname{var}(\mathrm{est})$ is the predicted variance of age (estimated likelihood curves). Thus, $r$ depicts the average magnitude of the relationship between the estimated and observed variance of combinations of a full set of scores. At an observed variance greater than estimated, the ad hoc correction widens the likelihood curves. At identical variances, the likelihood curves remain unaltered. If the 95\% prediction intervals obtained are realistic, then $95 \%$ of the age estimates should have an actual age which falls within this range (validation sample). This is known as coverage and was tested by investigating the percentage of individuals (validation sample) who fell within the $95 \%$ range.

The inaccuracy of the age estimates depicts the average magnitude of absolute error and was investigated by ( $\sum$ lestimated age - actual age $\mid$ ) $/ n$, where $n$ is the number of subjects in the validation sample, estimated age is the value of 
age which fulfill maximum likelihood and actual age refers to the chronological age at the time of examination. Bias represents the tendency of overestimation or underestimation of age and was investigated by ( $\sum$ lestimated age - actual age|)/n. Statistical analysis was performed using SPSS 19.0 software package for statistical analysis (IBM) except for the glm function, which was performed in the freely available statistical package " $R$ " [19]. When not described otherwise, a $p$-value of 0.05 was considered statistically significant.

\section{Results}

\subsection{Descriptive analysis}

An equal stage was assessed in the right and left maxillary third molars in $70.6 \%(316 / 425)$ of the males and $75.1 \%(322 / 429)$ of the female subjects. In the mandible, the figures were $77.4 \%(347 / 425)$ in males and $72.0 \%(309 / 429)$ in females, respectively. In the remaining 414 right-left third molar pairs, a maximum stage difference of 5 was found in one case. A stage difference of 1 was predominant in $73.91 \%$ of the cases. The remaining pairs ranged from 2 to 4 ( 2 stages in 68 pairs, 3 stages in 26 pairs and 4 stages in 13 pairs). In $52.4 \%$ of the male cases and $58.2 \%$ of the female cases, the right side was at a higher stage than the left, and this difference was significant for the mandible ( $p=0.004$, Wilcoxon Signed Rank test). The partial correlation coefficient (controlling for age) between third molars were higher within the dental arches than between (Table 2).

\subsection{Age estimates}

Age estimate curves (including maximum likelihood and prediction intervals) were obtained from stage 5 to stage 10 of UR, UL, LL and LR in males and females (reference sample). Fig. 1 illustrates stages of UR of 220 male subjects (reference sample).

As seen, the statistical approach of TA is less influenced by the age composition of the reference sample as compared to the means and standard deviations; at stage 5-9, the ages of maximum likelihood are younger than the comparable mean (Table 3 ). Similarly, likelihood curves were obtained for all third molars individually and multiplied to obtain estimates of age including a $95 \%$ prediction interval for every combination of a full set of scores (Fig. 2).

\subsection{Ad hoc correction}

In order to avoid too optimistic prediction intervals, the multiplied age estimate curves were corrected ad hoc. The validation sample was used for this purpose. Of the 1296 possible combinations of scores ( 6 possible stages raised to the power of 4 third molars), 69 and 88 were represented in males and females, respectively. The score 10101010 was predominant and represented $87 / 205$ (42.4\%) of male and 55/221 (24.9\%) of female cases. The second most representative combination was the score 5555 ( $8.3 \%$ males and $15.4 \%$ females). Due to a low number of cases, the

Table 2

Partial correlations controlling for age in males and females. All were significant (2tailed).

\begin{tabular}{lll}
\hline Third molars & Male, $n=425$ & Female, $n=429$ \\
\hline UR/UL & 0.777 & 0.857 \\
LR/LL & 0.904 & 0.855 \\
UR/LL & 0.670 & 0.588 \\
UR/LR & 0.688 & 0.615 \\
UL/LL & 0.661 & 0.671 \\
UL/LR & 0.678 & 0.691 \\
\hline
\end{tabular}

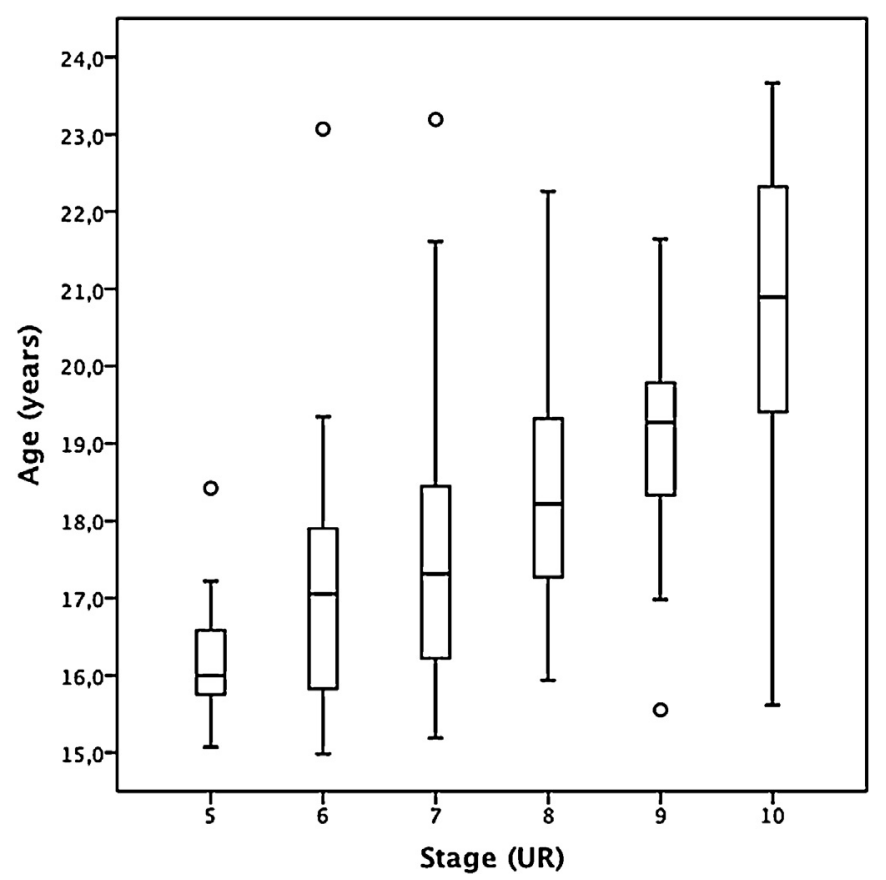

Fig. 1. Boxplots of the age distribution of stage 5-10 in the UR third molar for the male reference sample.

Table 3

Means and standard deviations as well as maximum likelihood and the $95 \%$ prediction interval using the statistical approach of TA of stage 5-10 in the UR third molar for the male reference sample.

\begin{tabular}{lllll}
\hline Stage UR & $\begin{array}{l}\text { Mean age } \\
\text { (years) }\end{array}$ & \pm 2 SD (years) & $\begin{array}{l}\text { Max. likelihood } \\
\text { of age (years) }\end{array}$ & $\begin{array}{l}\text { 95\% prediction } \\
\text { interval (years) }\end{array}$ \\
\hline 5 & 16.15 & $14.71-17.59$ & 15.00 & $15.00-17.62$ \\
6 & 17.19 & $13.95-20.43$ & 16.46 & $15.00-20.40$ \\
7 & 17.67 & $13.53-21.81$ & 17.13 & $15.00-21.09$ \\
8 & 18.35 & $14.81-21.89$ & 17.69 & $15.12-22.01$ \\
9 & 18.98 & $15.82-22.14$ & 18.34 & $15.63-23.42$ \\
10 & 20.78 & $17.12-24.44$ & 24.00 & $17.00-24.00$ \\
\hline
\end{tabular}

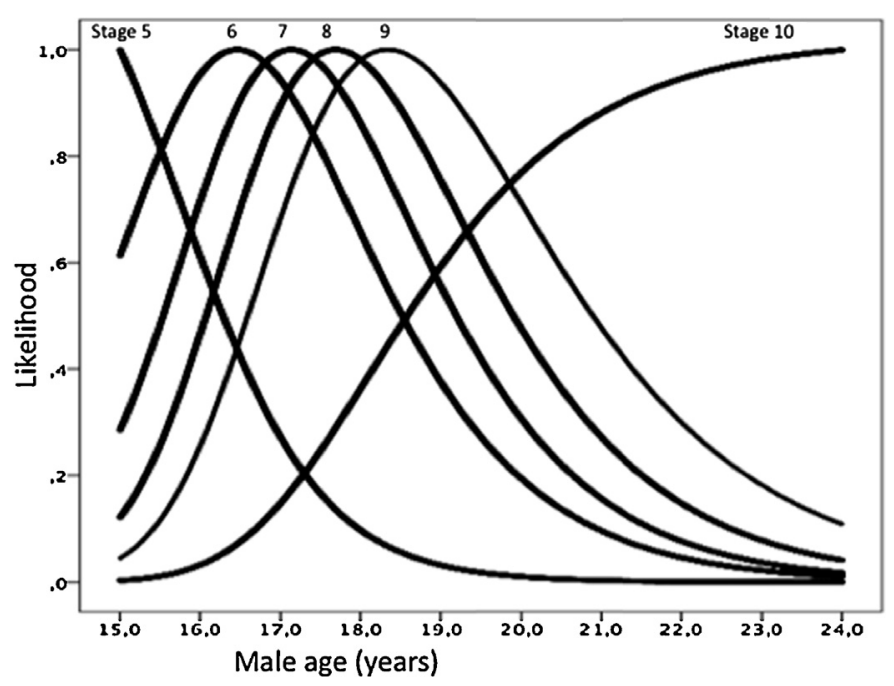

Fig. 2. Maximum likelihood curves of age based on TA for stage 5-10 in the UR third molar for the male reference sample.

total score (range 20-40) replaced the individual stage combination (Table 4).

Hence, var(obs) was based on the total scores (Table 4). Mean $r$, i.e. the relationship between the predicted and observed variance, 
Table 4

Distribution of individual scores in the four third molars and their added score (validation sample). For example, score 5555 equals a total score of $20(5+5+5+5)$.

\begin{tabular}{|c|c|c|c|c|c|c|}
\hline \multirow[t]{2}{*}{ Sex } & \multicolumn{5}{|c|}{ Score } & \multirow[t]{2}{*}{ Total score } \\
\hline & $n$ & UR & UL & $\mathrm{LL}$ & LR & \\
\hline M & 17 & 5 & 5 & 5 & 5 & 20 \\
\hline \multirow[t]{6}{*}{ M } & 4 & 5 & 5 & 6 & 6 & \\
\hline & 1 & 5 & 6 & 5 & 6 & \\
\hline & 1 & 5 & 6 & 6 & 5 & 22 \\
\hline & 1 & 5 & 7 & 5 & 5 & \\
\hline & 2 & 6 & 5 & 5 & 6 & \\
\hline & 6 & 6 & 6 & 5 & 5 & \\
\hline \multirow[t]{6}{*}{ M } & 3 & 10 & 10 & 9 & 9 & \\
\hline & 1 & 10 & 9 & 10 & 9 & \\
\hline & 1 & 10 & 9 & 9 & 10 & 38 \\
\hline & 1 & 9 & 9 & 10 & 10 & \\
\hline & 1 & 10 & 10 & 8 & 1010 & \\
\hline & 3 & 8 & 10 & 10 & & \\
\hline M & 87 & 10 & 10 & 10 & 10 & 40 \\
\hline $\mathrm{F}$ & 55 & 5 & 5 & 5 & 5 & 20 \\
\hline \multirow[t]{4}{*}{$\mathrm{F}$} & 4 & 5 & 5 & 5 & 6 & \\
\hline & 1 & 5 & 5 & 6 & 5 & \\
\hline & 2 & 5 & 6 & 5 & 5 & 21 \\
\hline & 4 & 6 & 5 & 5 & 5 & \\
\hline \multirow[t]{3}{*}{$\mathrm{F}$} & 1 & 5 & 6 & 6 & 7 & 24 \\
\hline & 10 & 6 & 6 & 6 & 6 & \\
\hline & 2 & 7 & 7 & 5 & 5 & \\
\hline \multirow[t]{7}{*}{$\mathrm{F}$} & 3 & 10 & 10 & 9 & 9 & 38 \\
\hline & 1 & 10 & 9 & 9 & 10 & \\
\hline & 1 & 9 & 10 & 10 & 9 & \\
\hline & 1 & 10 & 10 & 10 & 8 & \\
\hline & 1 & 10 & 10 & 8 & 10 & \\
\hline & 1 & 10 & 8 & 10 & 10 & \\
\hline & 2 & 8 & 10 & 10 & 10 & \\
\hline $\mathrm{F}$ & 55 & 10 & 10 & 10 & 10 & 40 \\
\hline
\end{tabular}

was 1.83 (range 1.19-4.57) in males and 3.40 (range 1.58-4.47) in females. The $95 \%$ prediction intervals were slightly wider in females than males. Hence, there was only a slight gain in using information from 4 third molars as compared to 1 in female cases. The estimated probability of an age below 18 years using a Chisquare distribution and 1 degree of freedom is $>2 \%$ and $<5 \%$.

\subsection{Accuracy}

At highly variable, high accuracy age estimates from third molar scores cannot be expected. Scatterplots showing individual age estimates versus actual age for the validation sample are seen graphically in Fig. 3, separated by sex. These plots represent raw data and provide a sense of inaccuracy and bias.

At $1.83 \pm 1.35$ years in males and $1.81 \pm 1.44$ years in females, mean inaccuracy is almost equal between sexes. The mean bias is $0.55 \pm 2.20$ years in males and $0.31 \pm 2.30$ years in females. Overall, the magnitude of this bias indicates that TA generally overestimates the chronological age in the living, slightly more in males than females. However, as illustrated, the direction of this bias is not constant, but tends to underestimate the chronological age of minors, especially for male cases, whereas the chronological ages of adults are slightly overestimated. Inaccuracy and bias are further described by age groups in Table 5 .

By defining the true positives as individuals where actual age and estimated age is equal to or above 18 years and the true negatives as individuals where actual age and estimated age is below 18 years, the diagnostic sensitivity of the age estimates were 0.824 in males and 0.814 in females, while specificity were 0.937 in males and 0.827 in females (Table 6). Hence, TA performs well at differentiating adults from minors, slightly better in males than females.

At $95.9 \%$, the percentage of observed female ages which fell within the 95\% prediction interval correspond to slightly overcompensated female likelihood curves. In males, the figure was 93.7\%, indicating slightly too optimistic prediction intervals.

\section{Discussion}

As confirmed by our results, the correlation, conditioning on age, between the 4 third molar stages are high [3,7,21], and stronger between molars located in the same arch (left/right) than opposite (maxillary/mandibular). Hence, more information about age may be anticipated from molars located in opposite arches than within. The slight left-right stage difference, although only significant in the mandible, may indicate that all four molars potentially carry information about age. The knowledge of which factors influence the rate of passage from one stage to the next may improve the possibility of attaining more accurate estimates of chronological age. However, Mincer et al. [7] showed that the use of two opposite located third molars as compared to one improved mean accuracy by only 0.1 year.

While a few studies have not found sexual dimorphism [5], most report a faster development of third molars in males than females [21-24]. Third molar development is generally completed 4-6 months earlier in males [7,21]. In Koreans, completion of the Demirjian stage $\mathrm{H}$ in male third molars has been reported up to 2 years ahead of females [12]. Our results suggest a higher female variability of age at each stage, which may be due to a slower development. This is a unique feature of the third molar compared to the sexual dimorphism in the other parts of the dentition and in bone development. Furthermore, as reflected by the sensitivity and specificity, the ability of TA to correctly discriminate between minors and adults was somewhat more accurate in our male subjects.

Genetic differences, such as ethnic origin, are considered important for the rate of third molar development. Retarded development has been found in Asians as compared to Caucasians and Blacks [25]. However, even though third molar development occurs at different rates between different ethnic origins, these differences are relatively small compared to the large inherent variability in third molar development. At an equal level of development, the maximum age difference reported between individuals originating from different countries is 14 months [26].

Using TA, mean inaccuracy of age estimates are in correspondence to previous published methods ranging from 1.13 to 1.97 $[5,11]$. A previous study reported an equal accuracy between a Bayesian approach and a classical regression model, but a lesser tendency for wrongfully classifying younger subjects as matured using Bayesian statistics [11]. This is in correspondence to the results of this study where only 4 in 63 male minors and 14 in 81 female minors, respectively, were wrongfully assessed as adults. As high accuracy age estimates cannot be anticipated due to the high inherent variability, it has been argued, that the unethical error of assessing minors as adults should be avoided [3,16]. Hence, the approach of TA follows this recommendation.

The model proposed by Thevissen et al. [11] showed slightly wider prediction intervals (97.2\%) as compared to the prediction intervals of this study. Although based on a relatively small sample with a narrow age range, the simple approach of correcting ad hoc seems meaningful and documents that TA is applicable to composite scores of third molar development. Future studies should be encouraged to investigate the applicability of TA even further. As the assumption of TA does not imply a complete set of estimated traits, the age estimation is based on the information available. In cases where information is lacking due to overlapping 

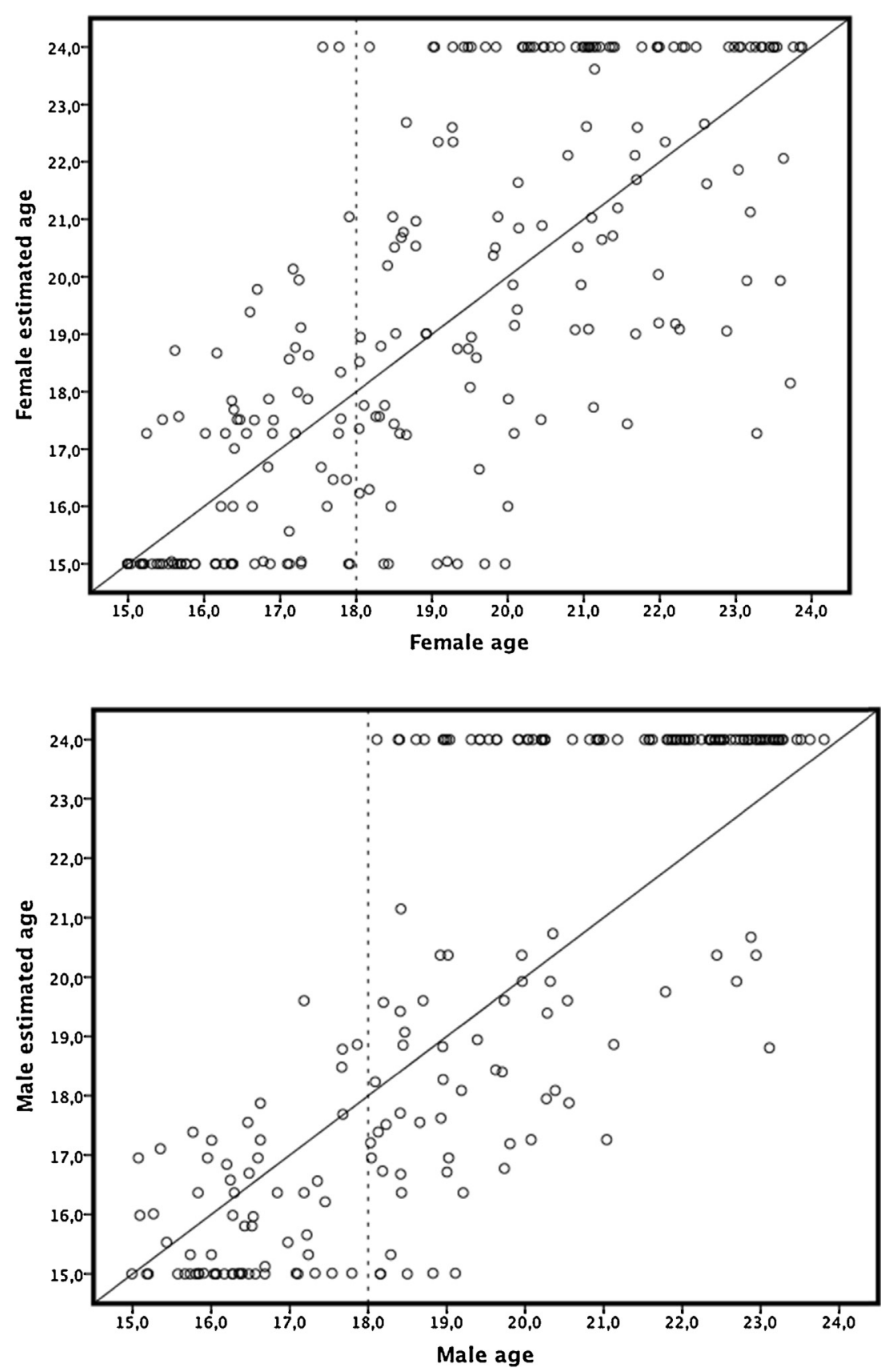

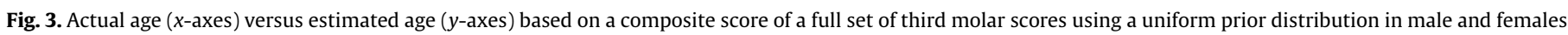
(validation sample). Solid line represents line of equality.

structures, developmental anomalies, or that the procedure has not been performed, this is an advantage. TA can be freely developed with the incorporation of more staging methods, indications of ethnic origin, as well as more traits, e.g. a radiograph of the left hand and wrist and MRIs of the clavicles. As such, available data based on other staging methods, e.g. Demirjian or the Olze method can be easily incorporated. The Olze method evaluates the soft tissue around the closed apex and may thus give additional information on the probability of having reached the 18year age limit [27]. However, methods must be reproducible in order to avoid an additional error due to the interobserver variability.

Although the assumption of conditional independence may hold a problem, no other statistical method currently combines the developing traits recommended by AGFAD and FASE, when performing age estimation in the living $[2,3]$. In order to develop a model which accounts for the correlations between developing traits, a very large number of radiographs are needed from young individuals of known ages. Such data are ethically difficult to obtain. However, the routine imaging performed prior to medicolegal autopsy may be used for this purpose [28].

As a juridical report of high standard, forensic age estimations must be performed and based on a solid scientific background which amongst other things includes a reproducible statistical procedure. Given that high-accuracy age estimates cannot be obtained, the variation and error of the age estimates should conversely be well described and reported in statements. The probability of the individual having reached certain age limits, e.g. 18 years, can be calculated. This has been proven useful for the legal system [2]. In addition, TA includes a graphical likelihood curve including maximum likelihood and a 95\% prediction interval. Such a graph may easier explain the probability function and the 
Table 5

Inaccuracy and bias values of error for a full set of third molars, male and female cases for each chronological age group (validation sample).

\begin{tabular}{|c|c|c|c|c|c|c|}
\hline Age group (years) & Sex & $n$ & Mean actual age (years) & Mean estimated age (years) & Inaccuracy years (SD) & Bias years (SD) \\
\hline \multirow[t]{2}{*}{15} & M & 18 & 15.57 & 15.70 & $0.81(0.51)$ & $0.13(0.97)$ \\
\hline & $\mathrm{F}$ & 25 & 15.45 & 15.45 & $0.74(0.76)$ & $-0.01(1.07)$ \\
\hline \multirow[t]{2}{*}{16} & M & 29 & 16.40 & 15.84 & $0.96(0.47)$ & $-0.56(0.92)$ \\
\hline & $\mathrm{F}$ & 29 & 16.48 & 16.64 & $1.19(0.71)$ & $0.17(1.40)$ \\
\hline \multirow[t]{2}{*}{17} & M & 15 & 17.42 & 16.57 & $1.57(0.81)$ & $-0.85(1.58)$ \\
\hline & $\mathrm{F}$ & 26 & 17.47 & 17.76 & $1.98(1.56)$ & $0.29(2.53)$ \\
\hline \multirow[t]{2}{*}{18} & M & 32 & 18.47 & 19.01 & $2.36(1.90)$ & $0.54(3.01)$ \\
\hline & $\mathrm{F}$ & 28 & 18.43 & 18.69 & $1.67(1.32)$ & $0.25(2.14)$ \\
\hline \multirow[t]{2}{*}{19} & M & 24 & 19.47 & 20.55 & $2.83(1.73)$ & $1.08(3.19)$ \\
\hline & $\mathrm{F}$ & 25 & 19.47 & 20.24 & $3.13(1.70)$ & $0.77(3.54)$ \\
\hline \multirow[t]{2}{*}{20} & M & 22 & 20.42 & 22.13 & $2.83(1.17)$ & $1.71(2.59)$ \\
\hline & $\mathrm{F}$ & 25 & 20.42 & 21.44 & $2.39(1.32)$ & $1.02(2.57)$ \\
\hline \multirow[t]{2}{*}{21} & M & 15 & 21.64 & 22.93 & $2.36(0.45)$ & $1.28(2.10)$ \\
\hline & $\mathrm{F}$ & 29 & 21.43 & 22.20 & $2.05(1.09)$ & $0.77(2.22)$ \\
\hline \multirow[t]{2}{*}{22} & M & 32 & 22.54 & 23.54 & $1.60(0.42)$ & $1.00(1.34)$ \\
\hline & $\mathrm{F}$ & 12 & 22.48 & 22.33 & $1.68(1.15)$ & $-0.15(2.09)$ \\
\hline \multirow[t]{2}{*}{23} & $\mathrm{M}$ & 17 & 23.27 & 23.70 & $0.94(0.89)$ & $0.43(1.24)$ \\
\hline & $\mathrm{F}$ & 21 & 23.42 & 22.68 & $1.48(1.71)$ & $-0.74(2.15)$ \\
\hline
\end{tabular}

Table 6

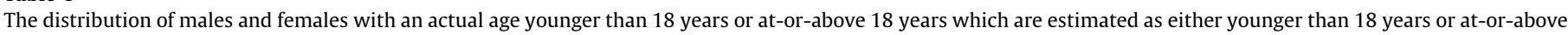
18 years.

\begin{tabular}{|c|c|c|c|c|}
\hline \multirow[t]{2}{*}{ Actual age } & \multicolumn{2}{|l|}{ Male, $n=205$} & \multicolumn{2}{|l|}{ Female, $n=221$} \\
\hline & Estimated age $<18(n)$ & Estimated age $\geq 18(n)$ & Estimated age $<18(n)$ & Estimated age $\geq 18(n)$ \\
\hline$<18$ & 59 & 4 & 67 & 14 \\
\hline$\geq 18$ & 25 & 117 & 26 & 114 \\
\hline
\end{tabular}

uncertainty of the age estimate to the recipients of the report, i.e. the legal system. Currently, only one reference sample of Korean origin has been evaluated using TA and as such, future studies should evaluate the application of TA on other ethnic origins, not least Caucasians.

\section{Conclusion}

This study evaluated the applicability of the reproducible and well-described statistical approach of TA on stages of third molar development in the age range 15.0-24.0 years on a cross sectional sample. At any combination of third molars, the described procedure provides age estimates (maximum likelihood) including a 95\% prediction interval as well as a probability of being above or below a certain age limit. While accuracy (the difference between estimated age and actual age) did not outperform other methods, this procedure provides age estimates which are unbiased. Hence, the risk of assessing a minor as an adult is minimalized. Furthermore, when corrected ad hoc, TA produces appropriate prediction intervals. As opposed to previous published methods, TA allows further scores to be added in a simple way. Thus, the future collection of data will allow expansion of this model to include bone age by a radiograph of the left hand and wrist and stages of clavicle development.

\section{References}

[1] K. Gunst, K. Mesotten, A. Carbonez, G. Willems, Third molar root development in relation to chronological age: a large sample sized retrospective study, Forensic Sci. Int. 136 (2003) 52-57.
[2] E. Cunha, E. Baccino, L. Martrille, F. Ramsthaler, J. Prieto, Y. Schuliar, N. Lynnerup, C. Cattaneo, The problem of aging human remains and living individuals: a review, Forensic Sci. Int. 193 (2009) 1-13.

[3] A. Schmeling, C. Grundmann, A. Furhmann, H.J. Kaatch, B. Knell, F. Ramsthaler, et al., Criteria for age estimation in living individuals, Int. J. Legal Med. 122 (2008) 457-460.

[4] S.J. AlQahtani, M.P. Hector, H.M. Liversidge, Brief communication: the London atlas of human tooth development and eruption, Am. J. Phys. Anthropol. 142 (2010) 481-490.

[5] H.M. Liversidge, P.H. Marsden, Estimating age and the likelihood of having attained 18 years of age using mandibular third molars, Br. Dent. J. 209 (2010) E13, http://dx.doi.org/10.1038/sj.bdj.2010.976.

[6] C.F. Moorrees, E.A. Fanning, E.E. Hunt, Age variation of formation stages for ten permanent teeth, J. Dent. Res. 42 (1963) 1490-1502.

[7] H.H. Mincer, E.F. Harris, H.E. Berryman, The A.B.F.O. study of third molar development and its use as an estimator of chronological age, J. Forensic Sci. 38 (1993) 379-390.

[8] S. Köhler, R. Schmelzle, C. Loitz, K. Puschel, Development of wisdom teeth as a criterion of age determination, Ann. Anat. 176 (1994) 339-345.

[9] J.L. Boldsen, G.R. Milner, L.W. Koningsberg, J.W. Wood, Transition analysis: a new method for estimating age from skeletons, in: R.D. Hoppa, J.W. Vaupel (Eds.), Paleodemography Age Distributions from Skeletal Samples, Cambridge, 2001, pp. 73-106.

[10] K. Haavikko, The formation and the alveolar and clinical eruption of the permanent teeth. An orthopantomographic study, Suom Hammaslaak Toim 66 (1970) 103-170.

[11] P.W. Thevissen, S. Fieuws, G. Willems, Human dental age estimation using third molar developmental stages: does a Bayesian approach outperform regression models to discriminate between juveniles and adults? Int. J. Legal Med. 124 (2010) 35-42.

[12] S.S. Lee, Y.S. Byun, M.J. Park, J.H. Choi, C.L. Yoon, K.J. Shin, The chronology of second and third molar development in Koreans and its application to forensic age estimation, Int. J. Legal Med. 124 (2010) 659-665.

[13] R.B. Bassed, C. Briggs, O.H. Drummer, Age estimation using CT imaging of the third molar tooth, the medial clavicular epiphysis, and the spheno-occipital synchondrosis: a multifactorial approach, Forensic Sci. Int. 212 (2011) 271-275.

[14] R.G. Aykroyd, D. Lucy, A.M. Pollard, T. Solheim, Technical note: regression analysis in adult age estimation, Am. J. Phys. Anthropol. 104 (1997) 259-265.

[15] E. Hillewig, J. Degroote, T. Van der Paelt, A. Visscher, P. Vandemaele, B. Lutin, L. D́Hooghe, V. Vandriessche, M. Piette, K. Verstraete, Magnetic resonance imaging 
of the sternal extremity of the clavicle in forensic age estimation: towards more sound age estimates, Int. J. Leg. Med. 127 (2012) 677-689.

[16] A. Schmeling, W. Reisinger, G. Gesserick, A. Olze, Age estimation of unaccompanied minors. Part I. General considerations, Forensic Sci. Int. 159 (Suppl 1) (2006) S61-S64.

[17] A. Demirjian, H. Goldstein, J.M. Tanner, A new system of dental age assessment, J. Hum. Biol. 45 (1973) 211-227.

[18] P.W. Thevissen, S. Fieuws, G. Willems, Third molar development: evaluation of nine tooth development registration techniques for age estimations, J. Forensic Sci. 58 (2013) 393-397.

[19] R Development Core Team, 2008, R: A Language and Environment for Statistical Computing, R Foundation for Statistical Computing, Vienna, Austria, ISBN 3 900051-07-0, URL http://www.R-project.org.

[20] L.W. Konigsberg, N.P. Herrmann, D.J. Wescott, E.H. Kimmerle, Estimation and evidence in forensic anthropology: age-at-death, J. Forensic Sci. 53 (2008) 541-557.

[21] G.Y. Levesque, A. Demirjian, R. Tanguay, Sexual dimorphism in the development, emergence and agenesis of the mandibular third molar, J. Dent. Res. 60 (1981) 1735-1741.
[22] V.J. Bhat, G.P. Kamath, Age estimation from root development of mandibular third molars in comparison with skeletal age of wrist joint, Am. J. Forensic Med. Pathol. 28 (2007) 238-241.

[23] P.W. Thevissen, S. Fieuws, G. Willems, Human third molars development: comparison of 9 country specific populations, Forensic Sci. Int. 201 (2010) 102-105.

[24] T. Uysal, Z. Sari, S.I. Ramoglu, F.A. Basciftci, Relationships between dental and skeletal maturity in Turkish subjects, Ang. Ortho. 74 (2004) 657-664.

[25] A. Olze, W. Reisinger, G. Geserick, A. Schmeling, Age estimation of unaccompanied minors. Part II. Dental aspects, Forensic Sci. Int. 159 (Suppl. 1) (2006) S65-S67.

[26] P.W. Thevissen, A. Alqerban, J. Asaumi, F. Kahveci, J. Kaur, Y.K. Kim, P. Pittayapat, M. Van Vlierberghe, Y. Zhang, S. Fieuws, G. Willems, Human dental age estimation using third molar developmental stages: accuracy of age predictions not using country specific information, Forensic Sci. Int. 201 (2010) 106-111.

[27] D. De Angelis, D. Gibelli, V. Merelli, M. Botto, F. Ventura, C. Cattaneo, Application of age estimation methods based on teeth eruption: how easy is the Olze method to use? Int. J. Legal Med. 128 (2014) 841-844.

[28] S. Tangmose, K.E. Jensen, C. Villa, N. Lynnerup, Forensic age estimation from the clavicle using 1.0T MRI-preliminary results, Forensic Sci. Int. 234 (2014) 7-12. 